\title{
Instrumentation and experimental investigation of industrial DC machines for condition monitoring purposes
}

\author{
Galina Mirzaeva, Terrence J.Summers and Robert E.Betz
}

\begin{abstract}
DC machines continue to be widely used in many applications including mining. No comprehensive condition monitoring tools for large industrial DC machines are available on the market. Recent advances in sensoring, data acquisition and signal processing technologies allow one to obtain real time chracteristics of DC machines directly related to their performance, such as flux density distribution inside the main air gap. This paper is based on a research project sponsored by industry partners that included construction of a test facility and an extensive experimental program with a view of development of a condition monitoring tool. The paper explains about the motor instrumentation, facility structure and tests performed to date. Based on the experimental results, a number of performance characteristics are derived in the paper including steady state, dynamic and digital drive related characteristics,as described in the paper. The findings of the paper are discussed with their potential application to the condition monitoring too development.
\end{abstract}

Index Terms-DC machines, DC motor drives, instrumentation, test facilities, testing, monitoring.

\section{INTRODUCTION AND MOTIVATION}

It is commonly believed that brushed DC machines are no longer in use or are in the process of being removed from the most of industrial applications. However, in some applications (such as traction, mining and submarine) DC machines do not only continue to be in service but also new DC machines are being produced. In Australian coal mining industry, for example, DC machines continue to be used with production critical equipment, such as electric rope shovels and draglines. These machines are used in open cut mining to dig and remove blasted overburden (with a big proportion of hard rock material) to expose a coal seam.

According to our survey, for a big mining company in Australia it is typical to have around $80 \%$ of DC machines in their motor fleet. This is partially due to vast experience with DC machines accumulated within the industry over the years. On the other hand, DC machines have proven to provide excellent dynamic performance and high robustness in particular demanding applications where high power end of electronic devices is combined with extremely dynamic load and harsh operation conditions. Brush and commutator maintenance seems to be a fair price for such performance characteristics.

The authors are with the School of Electrical Engineering and Computer Science, The University of Newcastle, Callaghan, NSW 2308, Australia (email: Galina.Mirzaeva@newcastle.edu.au Terry.Summers@newcastle.edu.au; Robert.Betz@newcastle.edu.au).

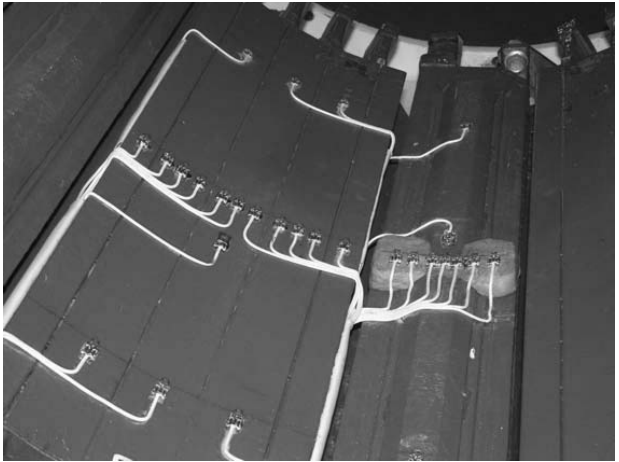

Fig. 1: Sensor positioning on one pole set

In the past years not much research has been done on DC machines while their operating conditions in the above mentioned applications have become much more demanding (due to continuous increase of bucket size and decrease of digging cycle). Previously obtained knowledge is insufficient to fully explain a range of issues involved in the commutator / brush wear in digging applications. Condition monitoring tools available for DC machines, to the authors' best knowledge, only cover mechanical vibration aspects of DC machines performance. No tools are available that would allow to predict the commutator / brush wear and to determine the maintenance needs of the machine.

On the other hand, in the past few decades a significant progress has been achieved in sensoring and data acquisition areas. It has become possible to measure and record, with high accuracy and resolution, characterics of DC machines that were only theoretically or indirectly obtained in the past, as presented in the classical text books. In particular, in the research project referred to in this paper direct measurements were obtained for flux density distribution inside the motor air gap.

The authors of this paper are currently involved in a research project supported by industry partners, which included construction of a test facility for full size (around 2000HP) industrial DC machines and an extensive experimental program under controlled steady state and dynamic conditions. The end purpose of this project is development of a DC Motor Duty Meter - a comprehensive condition monitoring tool that will relate on-line measurements of essential performance variables to the wear rate of the brush and commutator group. From the research prospective the project is interesting because: 
- Modern instrumentation can be applied and body of knowledge on DC machine performance can be extended;

- New techniques of condition monitoring will be applied resulting in development of a comprehensive tool for predictive monitoring of the DC machine performance;

- Potential improvements to performance and application of predictive maintenance would have high impact within Australian mining industry and other industries that use DC machines.

The rest of the paper is organised as follows. The next chapter explains about the motor instrumentation, facility structure and tests performed to date. Based on this data, performance of one of the test motors is analysed in detail, which includes its steady state, dynamic and digital drive related characteristics. These findings are discussed with their potential application to the condition monitoring tool development. The paper is concluded by the summary of the main ideas and contributions.

\section{Motor Instrumentation AND TESTING}

\section{A. Flux sensors selection and installation}

After an extensive appraisal of available on the market flux sensors, the Melexis MLX90251 Programmable Linear Hall Effect Sensor was chosen. It satisfied a number of requirements including: the measurement range (up to $1.8 \mathrm{~T}$ ); physical dimensions (less than $1 \mathrm{~mm}$ thick); bandwidth (up to $23 \mathrm{kHz}$ ); accuracy (12 bit); and second order thermal drift compensation for temperature ranging from $-40^{\circ} \mathrm{C}$ to $150^{\circ} \mathrm{C}$.

The flux sensors were calibrated using a specially constructed calibration rig that allowed to apply known flux density levels ranging from $-1 \mathrm{~T}$ to $1 \mathrm{~T}$. After calibration and before installation with the industrial motors, the sensors were tested with a laboratory scale DC machine.

Three pole sets of the same polarity in 6-pole industrial DC machines were instrumented with the flux sensors. The two test machines were of similar ratings $(2000 \mathrm{HP})$ but of different types, both commonly used with excavating machinery in mining. Fig. 1 illustrates sensor positioning on one of the pole sets of the test machine. The instrumentation challenges, such as reliable attachment of the sensors to the pole surfaces, minimisation of measurement and protection of the sensors during the motor assembling, were successfully met.

\section{B. Test facility}

The test facility included two DC motors connected back-toback, each with a digital control system, converter cabinet and power infrastructure as per a typical electric rope shovel installation. Block diagram of the experimental system is shown Fig. 2. At any moment of time one of the machines simulates a motor driving the bucket while the other performs the role of the corresponding dynamic load. Apart from the main pole and interpole flux sensors, each motor is instrumented by temperature sensors; armature current and voltage sensors; field current and voltage sensors; brush pigtail current sensors and a high frequency Rogowski coil sensor. Torque and speed transducers are installed on the common shaft.

The software of the experimental system consists of two parts illustrated by Fig. 3a and Fig. 3b, respectively: data

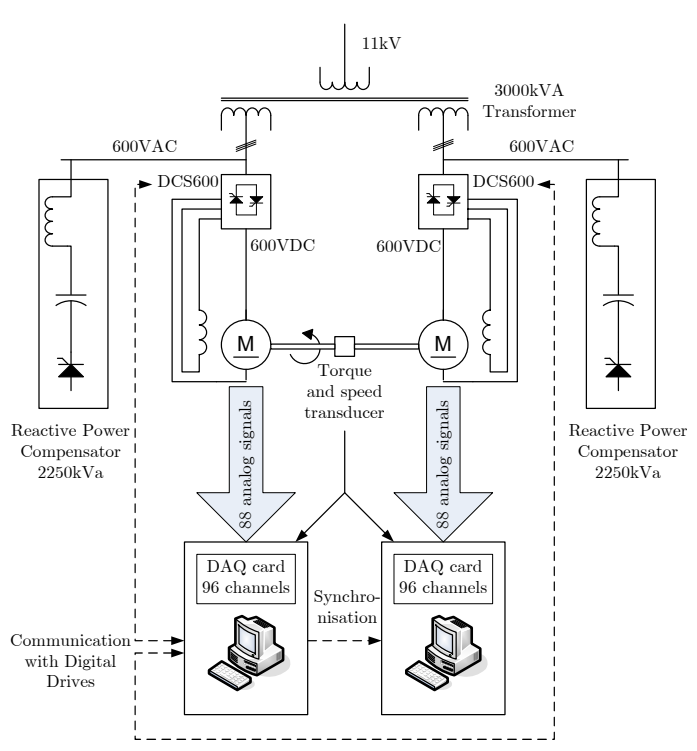

Fig. 2: Block diagram of the experimental system

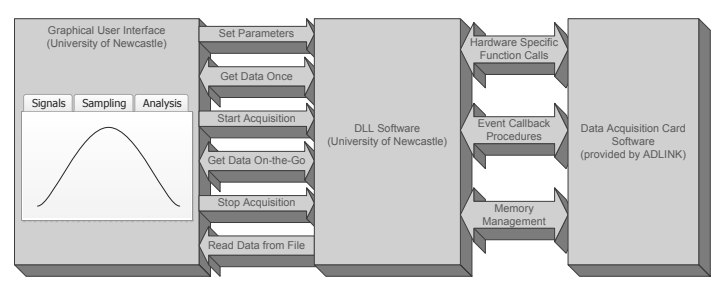

(a) Data acquisition software

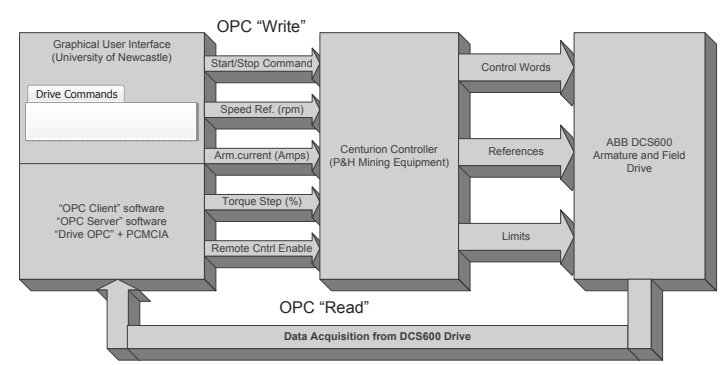

(b) Drive communication software

Fig. 3: Block diagrams of the system software

acquisition software that allows for fast sampling (up to $3 \mathrm{MHz}$ ) of a large number (up to 96) analog channels; and OPC-based digital drive communication software that allows to implement programmable test regimes. Commands to the drives are passed through a Centurion controller for protection purposes. More details on the facility structure and features can be found in [1].

\section{Experimental results}

Completed to date were initial short term tests where one of the parameters of interest was varied and the others were kept constant, which included:

- steady state tests at constant rotation speed and different armature current levels;

- steady state tests at constant armature current and different rotation speeds; 

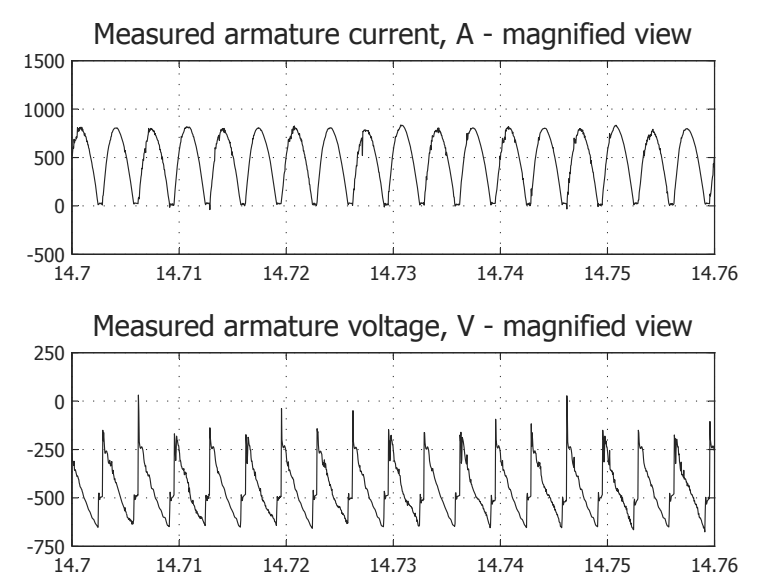

Measured main pole flux density, T - magnified view

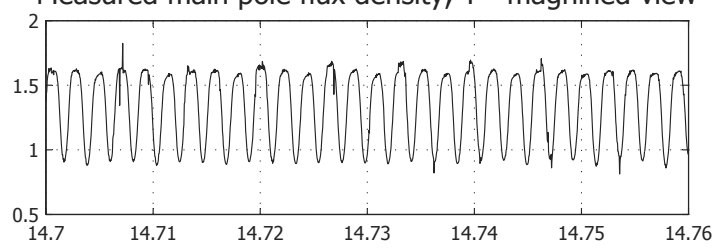

Measured interpole flux density, T - magnified view

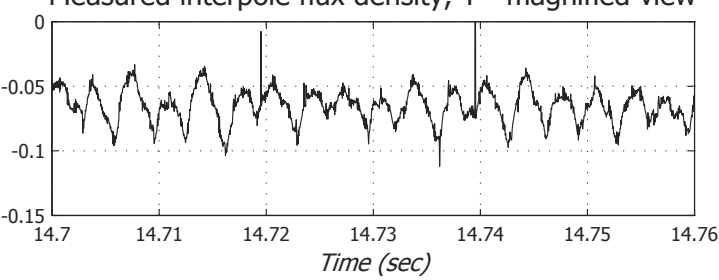

Fig. 4: Fragment of experimental data at 500A and 490rpm

- step changes of the armature current from zero to its rated value and back to zero at different "di/dt" settings of the digital drive;

- speed and current reversals at constant current limits.

The main objective of these tests was to obtain a thorough understanding of physical phenomena behind the brush and commutator wear. Longer term tests with physical simulation of digging cycles of the test machines are planned for the next stage of the project.

A magnified view of a fragment of experimental data is shown in Fig. 4, which corresponds to a steady state condition at $500 \mathrm{~A}$ and $490 \mathrm{rpm}$. The armature current and voltage have typical for SCR drives waveforms with $300 \mathrm{~Hz}$ ripple. Oscillations observed in the main pole flux signal are due to a slot effect, i.e. change of the air gap length as the armature slots pass by the flux sensor. Both $300 \mathrm{~Hz}$ ripple and slot effect can be observed in the interpole flux signal. Processing of experimental data to determine performance characteristics of one of the test machines is explained in the next chapter.

\section{DC MOTOR PERFORMANCE INVESTIGATION}

\section{A. Steady state performance}

Before studying the dynamic performance of the test motor it was important to make sure that its magnetic compensation is appropriately designed for steady state. The motor is equipped by interpoles and compensation windings to reduce the effects of armature reaction. Based on steady state measurements of flux densities in the air gap at different loads, the flux distribution curves of Fig. 5 was developed. These curves allowed to assess a number of aspects of the magnetic compensation, as explained below.

1) Brush alignment: If the brushes are aligned with the motor geometrical axes, then symmetrical $\pm I_{a}$ load conditions would correspond to symmetrical field measured in the geometrically neutral position, $\mp B_{i p}$ or $\pm B_{i p}$, depending on motoring or generation mode. It can be seen from Fig. 5 that, for example, for the armature current $I_{a} \approx \pm 2000$ A the flux density under the middle of the interpole was $B_{i p} \approx \mp 0.24 \mathrm{~T}$, which indicated the appropriate brush alignment.

2) Compensation winding performance: The main purpose of the compensation windings is to compensate for the armature reaction under the main poles at any load. A reasonably flat shape of the flux density under the main pole seen in Fig. 5 showed that the compensation winding served its purpose. A saw-tooth component on top of the average horizontal level observed in Fig. 5 corresponds to discretization effect (finite number of turns) of the compensation winding.

3) Interpole performance: The interpoles are used to compensate, at any load, for the armature reaction in the commutation zone and to provide a load dependent field that assists commutation (in other words, interpoles should slightly overcompensate for the armature reaction). From Fig. 5 it can be seen that the field under the interpole is of the opposite polarity to the armature current and of notable magnitudes that are roughly proportional to the armature current magnitudes. It is not a trivial task to determine whether the flux magnitudes measured under the interpole are appropriate to the load.

To do that we recall from the classical commutation theory (see, for example, [2]) that for linear commutation the emf produced by the total external field in the commutation zone should match the reactive emf of the coil undergoing commutation:

$$
2 n_{c} l_{c} v_{a} B_{e x t}=L_{e q} \frac{2 i_{a}}{T}
$$

where $n_{c}$ and $l_{c}$ are the number of turns and the length of the commutating coil, respectively; $v_{a}$ is linear speed of the armature periphery; $B_{\text {ext }}$ is the flux density of the external field in the commutation zone; $L_{e q}$ is equivalent inductance of the commutating coil taking into account mutual coupling with other coils; $i_{a}$ is current in one current path of the armature winding (current that is actually being commutated in the coil); and $T$ is commutation period.

It follows from (1) that for linear commutation the field in the commutation zone should be proportional to the armature current. The proportionality coefficient can be found from (1) based on known parameters of the DC machine. In case that the thickness of the brush $b_{b r}$ is bigger than the thickness of one commutator segment $b_{\text {com }}$, the current reversal in the coil takes a proportionally longer time than for $b_{b r}=b_{c o m}$. In other words, the commutation period equals to $b_{b r} / v_{c}$ where $v_{c}$ is linear speed of the commutator periphery.

Another effect of a thicker brush is that several coils undergo commutation simultaneously, which results in an 


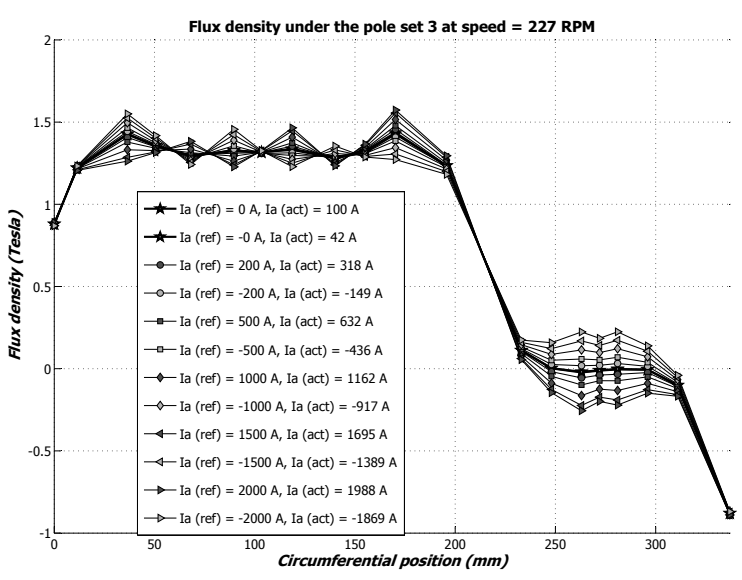

Fig. 5: Flux densities in steady state at different loads

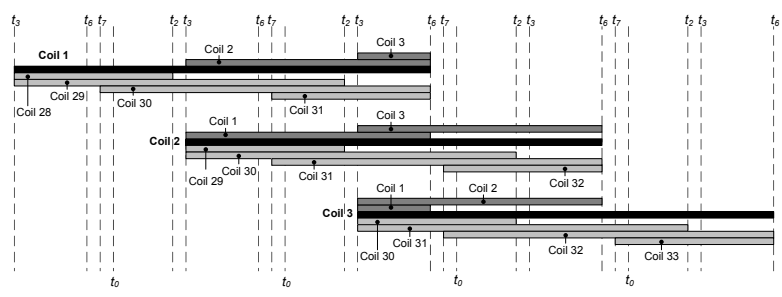

Fig. 6: Coil mutual inductance consideration

increased equivalent inductance $L_{e q}$ compared to self inductance of one coil $L_{c}$. To accurately account for this effect, commutation timing was considered beyween a number of coils, as illustrated in Fig. 6. It was further considered that some coils which commutation overlaps in time are coupled with each other by one side and some coils - by both sides laid in the same armature slot. It was found for the test machine that $L_{e q} \approx 2.4 L_{c}$.

With all these numbers substituted in equation (1), for linear commutation the test machine the following relation should stand: $B_{\text {ext }} \approx 1.2 * 10^{-4} I_{a}$. Now, from the experimental data illustrated by Fig. 5, it was found that the flux density measured under the centre the interpole $B_{i p}=B_{\text {ext }} \approx$ $1.3 * 10^{-4} I_{a}$. Although from the first glance at Fig. 5 it looks like the field under the interpole is too strong, the above calculations have shown that the interpole strength is very close to its optimal value for linear commutation.

\section{B. Dynamic performance}

The rate of change of the armature current is limited by the drive "di/dt" setting. A typical value for this setting in digging applications is $3 \%$ of the rated current per msec. Given that the internal commands in the DSC600 drive are processed every $2 \mathrm{msec}$, it is clear that the rate of change of the armature current cannot be controlled precisely. A number of tests with different "di/dt" settings were performed where a step change of the armature current from zero to its rated value and back to zero was commanded. It was experimentally found that the actual rate of change of the armature current was approximately a

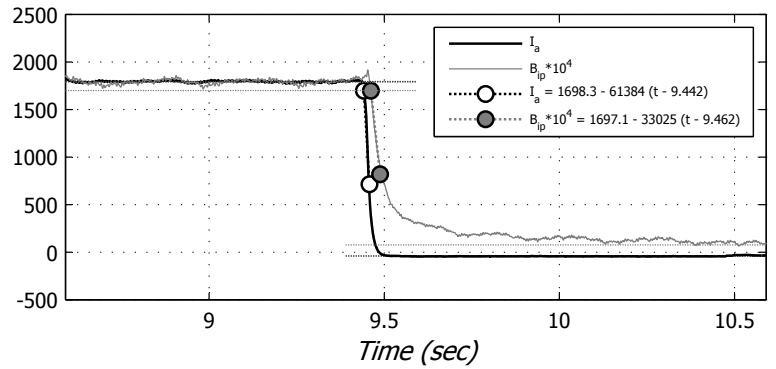

Fig. 7: Dynamics of armature current and flux density in commutation zone under a step change

half of the drive "di/dt" setting on the rising edge of the step and slightly bigger than a half on its falling edge.

More importantly, it was experimentally found that the field in the commutation zone changes at even slower rates and with notable delays as compared to the armature current changes. This is illustrated by Fig. 7 which shows in one plot a fragment of experimental data for the armature current and the flux density under the middle of the interpole as the armature current drops from its rated value to zero. It can be seen from Fig. 7 that the flux density in the commutation zone starts to drop $20 \mathrm{msec}$ later than the armature current. At the speed of $227 \mathrm{rpm}$ at which the measurement was taken the commutation repeats itself every $1.5 \mathrm{msec}$, which means that as many as 13 commutations occur before the magnetic compensation starts moving towards its appropriate level. All this time the motor remains over-compensated.

Similarly, when the armature current ramps up, a delay in the magnetic compensation for a few commutation periods results in under-compensated conditions. The most likely reason for the observed delays in the flux dynamics are Eddie currents in the interpole pole piece.

\section{SCR drive issues}

1) Commutation of pulsating current: Due to application of the SCR drive, the actual armature current and related waveforms have a $300 \mathrm{~Hz}$ ripple on top of DC values. This can be clearly seen from the fragment of the experimental data in Fig. 4. The presence of the ripple means that even in steady state conditions of commutation can be different for each individual coil. Compare Fig. 8a corresponding to a pure DC supply to Fig. 8b corresponding to the SCR drive supply for the DC machine. In the latter plot the commutating current deviate from and join into the pulsating waveform at different moments of time relative to the period of $300 \mathrm{~Hz}$ ripple.

The plots in Fig. 8 were obtained by using a dynamic model of commutation. This model described in detail in [3] is based on solving the system of differential equations for commutation currents $i_{k}$ :

$$
\frac{d i_{k}}{d \tau}=\frac{\rho_{b r} l_{b r}}{L_{e q_{k}} T}\left(\frac{i_{k-1}-i_{k}}{S_{b r_{k}}(\tau)}-\frac{i_{k}-i_{k+1}}{S_{b r_{k+1}}(\tau)}\right)+\frac{1}{L_{e q_{k}} T} e_{m}(\tau)
$$

where $\rho_{b r}$ and $l_{b r}$ are the brush resistivity and length, respectively; $S_{b r_{k}}$ is the variable contact area between the brush and commutator segment $k ; \tau$ as time variable normalised to the 


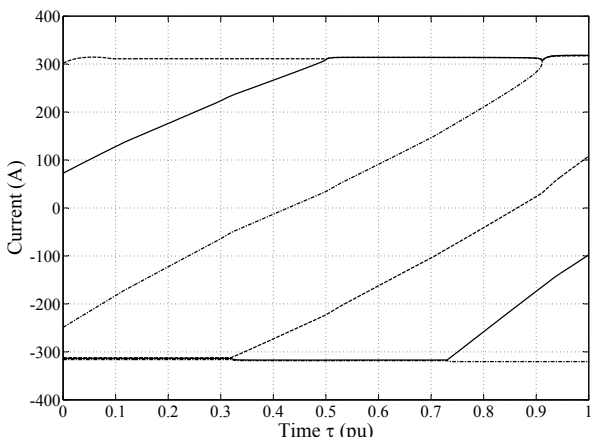

(a) Commutation of pure DC current

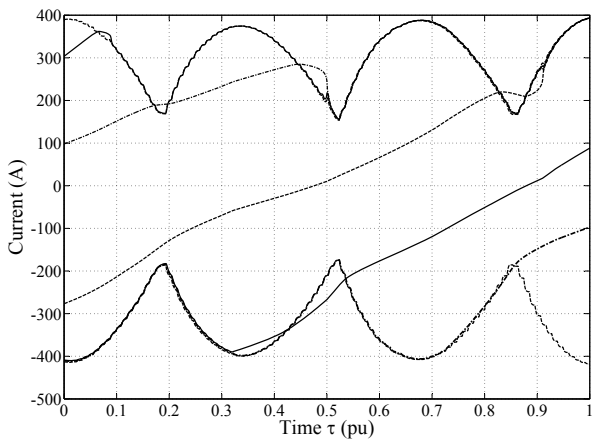

(b) Commutation of pulsating current

Fig. 8: Commutation of current from a DC source versus SCR

commutation period $\tau=t / T$; and $e_{m}(\tau)$ is the "motional" emf corresponding to the left hand side of equation (1). Each current $i_{k}$ equals to $-i_{a}$ before and $i_{a}$ after the commutation is completed. Current $i_{a}$ in one path of the armature winding, as well as the motional emf $e_{m}(\tau)$, are found from the experimental data for the armature current and the flux density under the interpole. The model is further enhanced by applying a non-linear model for the brush resistivity, which results in a non-linear brush voltage drop, as explained in [4].

From comparison of the commutation dynamics for the pure DC current and for the pulsating current in Fig. 8 it clearly follows that commutation conditions for a DC machine fed from an SCR drive are more difficult than for the same machine fed from a pure DC source, which may translate into faster wear rates of the brush and the commutator. On the other hand, commutation of the pulsating current in "steady state", given that the magnetic compensation is adequate, goes without major discontinuities, as can be appreciated from Fig. 8b. In addition to that, commutation conditions are different for different rotation speeds, which is investigated in the next subsection.

2) Spectral analysis: To compare performance of the DC machine performs at different speeds, experimental data was collected for steady state conditions at constant load (1000A) and different rotation speeds. Fifteen different speed values were so selected that they evenly covered the operation range between 80rpm and 950rpm and included some particular speeds of interest (for example, speeds corresponding to $300 \mathrm{~Hz}$ ripple and its harmonics). To achieve a better accuracy of spectral estimations, an average of seven FFTs was
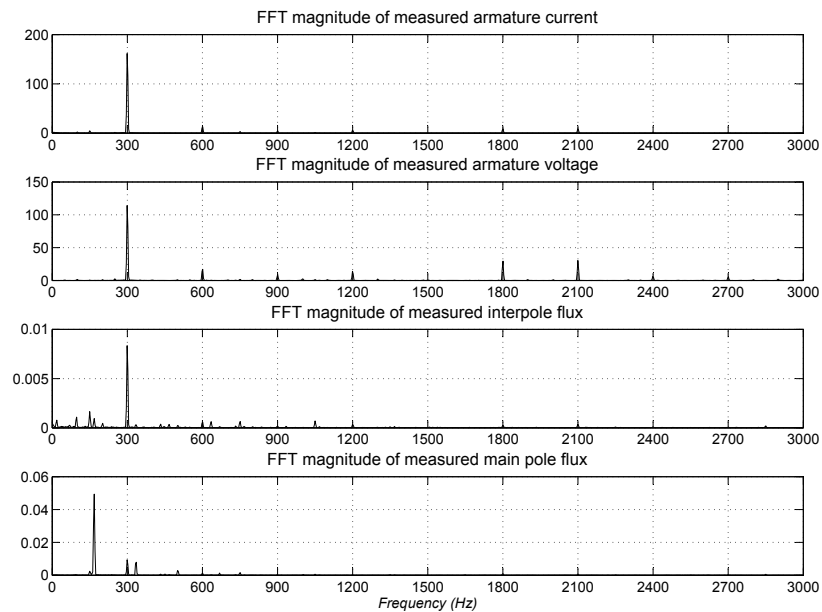

Fig. 9: Spectra of signals at rotation speed of 171rpm

calculated for each data series, each FFT being based on 8192 points of data with mean values being subtracted and Hamming window being applied [5].

Example spectra for concurrently sampled armature current, armature voltage, flux densities under the main pole and under the interpole are shown in Fig. 9, which corresponds to the experimental data collected at $171 \mathrm{rpm}$. Some interesting observations can be made with the help of Fig. 9. As was expected, the $300 \mathrm{~Hz}$ component is strongly pronounced in the armature current and armature voltage spectra. Its higher harmonics are first reducing in magnitude but then increasing again around $1.8--2.1 \mathrm{kHz}$. This effect was related to ringing observed on a magnified view of voltage, at the thyristors turnon moments. This ringing could be due to wave reflection in the drive-to-motor cable, or to a resonance in a thyristor snubber circuit, which needs a further investigation.

Another group of frequency components is associated with the armature slot effect. Conductors of the armature winding is laid in discrete slots. When a slot passes by a stationary installed main pole or interpole flux sensor, the effective air gap increases and its permeance drops. The frequency of the slot effect is given by:

$$
f_{s l}=n_{r p m} \frac{N_{s l}}{60}
$$

where $n_{r p m}$ is rotational velocity in RPM; $N_{s l}$ is the number of slots.

As our primary concern was the quality of commutation, comparison between the spectra for the armature current $I_{a}$ and the flux density $B_{i p}$ under the interpole (in the middle of commutation zone) was of particular interest. This comparison was done for all speeds of rotation and for a number of spectral components including:

- $300 \mathrm{~Hz}$ ripple and its harmonics. These components were consistent and of approximately proportional magnitudes between the two spectra (of $I_{a}$ and $B_{i p}$ ) at all rotation speeds, which suggested that these components in $B_{i p}$ were caused by their their presence in $I_{a}$.

- Frequency $f_{s l}$ of the slot effect and its harmonics. These components were not observed in the armature current 
spectrum but were strongly pronounced in the spectrum of the interpole flux density affected by the slot effect.

- Beat frequencies between the above two. Since the interpole flux signal contains both drive related $(300 \mathrm{~Hz})$ and rotation related $\left(f_{s l}\right)$ components, it is reasonable to expect that spectral components at $\left(f_{s l} * k \pm 300 * m\right)$ would appear in the interpole flux spectrum. The beat frequencies $\left(f_{s l}-300\right)$ and $\left(f_{s l}-600\right)$ were found to be of particular importance. They appear in the low frequency range, travel as the motor rotational velocity changes, and may excite mechanical vibrations of the motor frame and brush holders. It was observed experimentally that at some rotational velocities low frequency mechanical vibrations become evident, which then disappear as the velocity changes.

In summary, spectral analysis of the steady state data at different speeds showed presence of certain frequency components and some interesting effects with potential implications for commutation, which needs a further detailed investigation.

\section{CONTRIBUTIONS TO CONDITION MONITORING AND CONCLUSIONS}

Basic mechanisms of the brush and commutator wear are well known. As explained in [6], the main parameters that determine the brush life are: presence of commutator film, temperature, current density dependent on the load, speed, humidity and mechanical vibration. The associated wear mechanisms can be experimentally quantified. Depending on a particular combination of the factors the rate of brush and commutator wear can be estimated and applied in a predictive monitoring tool.

The above approach forms the basis for development of the condition monitoring tool in the research project described in this paper. The new contributions of the paper are seen in that:

- It proposes a new and effective way of estimation of the DC motor condition - via on-line measurement of flux density inside the air gap in the commutation zone. This allows to compare the magnetic compensation at any point in time to its desired value, to measure mechanical vibrations, electrical resonances and other disturbances to commutation.

- The paper proposes "dynamic compensation" as a factor to be included in the DC motor performance model for condition monitoring. Under dynamic changes the effect of magnetic compensation can be delayed resulting in poor compensation for a number of commutation periods. In dynamic applications like digging this factor may play an important role in brush and commutator wear. The proportion of time that the motor spends in dynamically uncompensated state can be measured on-line, using the technique explained in this paper, and included in a DC motor life prediction model.

- The paper further explores the effects of the SCR drive on the DC motor commutation. Strongly pronounced spectral components in the measured signals need further investigation with regard to their consequences for commutation and motor life.
- Based on the paper findings, potential improvements in motor design for mitigation of the undesirable effects may result.

In conclusion, the initial stage of the DC motor experimental testing program showed a number of interesting results. They contributed to the body of knowledge, opened new opportunities for condition monitoring of DC machines in general and supported viability of the DC Motor Duty Meter concept. Future work includes long term tests where repeated load cycles representing digging conditions are applied and deterioration of the brush and commutator is measured.

\section{REFERENCES}

[1] G. Mirzaeva, R. Betz, T. Summers, and I. Marxsen, "Development of a unique dc motor test facility in the hunter valley," in Proc. of the Australian Mining Technology Conference. 16-18 September 2008, Twin Waters, QLD, Australia, 2008, pp. 95-106.

[2] A. E. Clayton, The performance and design of direct current machines, 3rd ed. London: Sir Isaac Pitman and Sons Ltd, 1969.

[3] G. Mirzaeva, R. Betz, and T. Summers, "Evaluation of current density in $\mathrm{dc}$ motor brushes for mining machines based on air gap field measurement," in Proc. of the Industry Applications Society Annual Meeting. 4-8 October 2009, Houston, TX, 2009, pp. 1-8.

[4] Z. Qingliang, T. Ueno, and N. Morita, "Basic studies for accurate commutation analysis which enables commutation spark energy estimation," in 18th International Conference on Electrical Machines and Systems, 2008. ICEMS 2008 Conference Record of the, 17-20 October 20082008 , pp. $42-47$.

[5] A. Oppenheim and R. Schafer, Discrete-Time Signal Processing. Prentice-Hall, 1989.

[6] R. Hamilton, "Dc motor brush life," IEEE Transactions on Industry Applications, vol. 36, no. 6, pp. 1682-1687, 2000.

Galina Mirzaeva received M.E (1990) and PhD (1997) from the South Ural State University, Russia. In 1993-1998 she worked with the Makeev National Rocket Centre, Russia. In 2004-2009 she was a Research Academic with CRC Mining at the University of Newcastle, Australia. Since 2009 she is a Senior Lecturer with School of Electrical Engineering and Computer Science of the same University. Her research interests include control and dynamic performance of $\mathrm{AC}$ and $\mathrm{DC}$ machines and various aspects of power electronics and autonomous power generation. She is a member of Industrial Drives Committee and the Chair of Mining Industry Committee of the IEEE Industry Applications Society.

Terrence J. Summers received B.E (2001) and PhD (2007) degrees in electrical engineering from the University of Newcastle, Australia. He is currently a Lecturer with School of Electrical Engineering and Computer Science of the same University. His research interests are in electrical drives and power electronics. He is a member of the Industrial Drives Committee and Mining Industry Committee of the IEEE Industry Applications Society.

Robert E. Betz received the B.E, M.E and $\mathrm{PhD}$ degrees in electrical engineering all from the University of Newcastle, Australia in 1979, 1982 and 1984 respectively. His research interests are in various aspects of the control of AC machines, with particular emphasis on high performance control of induction and synchronous reluctance machines. More recently he has also been working in the power electronics area on the multi-level cascaded H-bridge STATCOMs. He is currently the Ampcontrol Professor of Power Engineering at the University of Newcastle, and is the Chief Technical Consultant of ResTech Pty Ltd, a joint venture company between Ampcontrol Pty. Ltd. and the University of Newcastle, Australia. Professor Betz is a member of the Industrial Drives Committee and Mining Industry Committee of the IEEE Industry Applications Society. 